\title{
Trialling technologies to reduce hospital in-patient falls: an agential realist analysis
}

\section{Abstract}

This paper analyses the 'failure' of a patient safety intervention. Our study was part of an RCT of bed and bedside chair pressure sensors linked to radio pagers to prevent bedside falls in older people admitted to hospital. We use agential realism within science and technology studies to examine the fall and its prevention as a situated phenomenon of knowledge that is made and unmade through intra-actions between environment, culture, humans and technologies. We show that neither the intervention (the pressure sensor system), nor the outcome (fall prevention) could be disentangled from the broader sociomaterial context of the ward, the patients, the nurses and (especially) their work through the RCT. We argue that the RCT design, by virtue of its unacknowledged assumptions, played a part in creating the negative findings. The study also raises wider questions about the kind of subjectivities, agencies and power relations these entanglements might effect and (re)produce in the hospital ward.

\section{Keywords}

RCT; bed sensor; agential realism; fall; elderly patients

\section{Authors}

Professor Stephen Timmons, Professor of Health Services Management, Nottingham University Business School

Dr. Paraskevas Vezyridis, Assistant Professor in Human Resource Management, Nottingham University Business School

Professor Opinder Sahota, Department of Healthcare of Older People, Nottingham University Hospitals NHS Trust 


\section{Introduction}

In this paper we take an agential realist approach (Barad 2007) to understanding how and why a patient safety technology 'failed'. Our study was part of an RCT of pressure sensors linked to radio pagers being trialled to prevent bedside falls in older people admitted to hospital (Sahota et al. 2013). The fact that this technology was the subject of an RCT is of central importance in understanding this failure. In the context of a RCT, the identification of research participants and the allocation of these patients to the trial groups can be problematic since the assessments used to identify patients at risk have limited specificity (Shorr et al. 2012) and it is impossible to blind nurses (or patients) with 'dummy' sensors (Sahota 2009). The RCT and the sensor system are intertwined practices and here we offer both a critique of the assumptions about how RCTs work (e.g. that it is impossible to intervene in complex care practices and yet test interventions in a controlled mannernurses, patients, clinical practices, physical layout) and that technologies 'do not do' what their designers think they will do - while they have to assume responsibility for caring for its use (batteries etc.).

Preventing a fall is, in fact, a multi-factorial collective effort of a range of factors that do not solely reside in either the technological, organisational, or human domains. Thus, we studied how the trial of a technology is enacted, without missing these invisible actors from examination. We did not assign a priori responsibilities and accountabilities to either nurses' individual awareness of risky body movements or the technology's affordances (Horton et al. 2017). For us, the failure to prevent in-hospital falls is not to be found in either the staff, the technology, the mode of observation, or any other actor entangled within this phenomenon. The answer is to be found somewhere within their 
complex and dynamic relationships that configure the sociomaterial phenomenon of falls prevention. By moving away from individualised understandings of knowledge and action to "achievement as a collective flow" (Wohlwend et al. 2017: 459), we provide more 'situated' and less deterministic explanations of why the production of 'evidence' in RCTs (Savransky and Rosengarten 2016 ) is often detached from the wider practice of healthcare, leaving the technologically-mediated prevention of falls trialled in isolation.

\section{The technology-in-trial}

A pressure sensitive pad is placed under the bed mattress and bedside chair cushion, and it transmits an alert to a central monitor in the ward if the pressure on the plate is reduced. We will use 'sensor' for both the bed and bed chair sensors. The central monitor then sends a message to a pager carried by a nurse, who is alerted that the patient has got out of bed or their bedside chair. The member of staff can then respond appropriately. A pilot study carried out in the same setting (Till et al. 2005) had shown encouraging results for reducing falls with this technology. This RCT was conducted in inpatient care of older people wards in a UK hospital. The results of the RCT were that sensors linked to radio pagers do not reduce in-patient bedside falls or time to first bedside fall, and are not cost-effective (Sahota et al. 2013). The data we are analysing in this paper are from a qualitative project nested within the RCT.

The intervention being evaluated in the RCT needs to be understood in the context of fall prevention more generally on the wards studied. A number of processes were already in place to address this risk. A comprehensive, documented assessment of falls risk was undertaken for each patient on admission, though it is not clear that assessment alone is effective in preventing falls (Meyer et al. 2009). Indeed, nurses' clinical judgement has been shown to be superior to structured assessment tools in predicting risk of falls (Vassallo et al. 2008), and the issue of clinical judgement versus the assessment of risk more 'mechanically' is one to which we shall return. Other preventative measures range from the simple (ensuring patients have suitable footwear and vision correction) through 
equipment (the use of bed rails or ultra-low beds) or physiotherapy interventions, to pharmaceutical treatments (for instance, to address postural hypotension). Nurses have very clear notions of what aspects of their work they consider to be the most important ; typically direct, hands-on patient care, and work that is a lower priority, usually tasks that have been imposed by the organisation, or paperwork (Timmons, 2003). These ideas are deeply-seated in the informal culture of nursing (Allen 1997)

\section{The agential realism of a technology-in-trial}

We are using a performative perspective situated in Science and Technology Studies (STS) (Latour 2005, Mol 2002, Barad, 2007). The main insight of this tradition is that technologies are not neutral objects, and that they have an active existence, and meanings, within the sociomaterial world. A Cartesian ontological perspective separates the social from the technical as independent entities, each with its own distinct properties (Riemer \& Johnston, 2014) residing in an objective world. In that case, nurses and the bed sensor system are actors which can only interact and, thus, our analysis would have focused more on their properties and less on their relationship, explaining (deterministically) why this particular technology failed. In contrast, a performative approach seeks to move beyond both technological and social determinism. Technology is analysed in a dialectical relationship with its users, in context (Latour 2005). The meaning and use of a technology are not defined by its designers, but emerge through a process of adaptation and reframing as it is implemented (or not). As Webster (2002: 444, italics in original) says "technologies are only really successful when they make sense within the existing social relations within which they are to function, suggesting the crucial role played by the translation and even reinvention of technologies into everyday contexts of use". This is very different to the assumption inherent (but never really made explicit) in an RCT, where interventions are seen as being epistemologically neutral and ontologically independent. As Montgomery and Pool (2017: 59) concluded: 
'Study subjects are not located in the social vacuum which the RCT idealizes; they are situated at the intersection of other forms of inclusion and exclusion, both locally and globally...These more permanent social structures need to be taken into consideration in the design of research, even where that research is testing a biological "magic bullet"'.

Will (2009) has shown the methodological limits of RCTs as tools to produce "proofs of protocol" to manage uncertainty in healthcare "organizing an RCT requires careful negotiations between the requirements of rigour and relevance" (Will 2007: 87). As Will and Moreira (2016: 10) say:

[An] ethnographic perspective provides a space to explore the complex and contingent resolutions of the debates that follow trials, probing the investments of different collectives in particular forms of evaluation, and the ways in which scientific and social decisions are co-produced in situations of interpretative flexibility left by the clinical trial. Here the work of doing trials, or 'research', is not imagined as somehow distinct from clinical work, or 'practice',."

With agential realism, Barad $(2003 ; 2007)$ invites us to study the social and the material by taking for granted a reality that is fluid, dynamic, complex and entangled :

'Reality is composed not of things-in-themselves or things-behind-phenomena but things-inphenomena. The world is a dynamic process of intra-activity and materialisation in the enactment of determinate causal structures with determinate boundaries, properties, meanings, and patterns of marks on bodies. (...) It is through specific agential intra-actions that a differential sense of being is enacted in the ongoing ebb and flow of agency. ... it is through specific intra-actions that phenomena come to matter - in both senses of the word' (Barad 2007: 140).

The focus is on the relationships that enact sociomaterial phenomena. Both matter and meaning are equally important and are co-constituted non-hierarchically, acquiring their agency and importance through their entanglement and intra-action (Barad 2007). Agency is not a property of separated entities but emerges from (and is distributed over) continuously negotiated relationships where actors are brought together and 'intra-act' (Barad 2007) (rather than inter-act) in co-constitutive and 
mutually responsible ways for the matter to be produced, whether that is discourses, materialities and/or subjectivities:

'The concept of intra-action reframes materiality from design affordance to a cycling interplay produced by the physicality, fluidity and messiness of entangled bodies, things and places' (Wohlwend et al. 2017: 447).

Individual agencies, causes and effects, or subject-object dichotomies no longer have a place in the study of the phenomenon. It "is through specific intra-actions that the boundaries and properties of the "components" of phenomena become determinate and that particular embodied concepts become meaningful" (Barad 2003: 815). Here, any body movements that the sensor detects and the RCT measures, do not pre-exist. Times and spaces of practices in acute care wards are not necessarily linear and geographically specific, but simultaneous in their enactment and agency. All will materialise, acquire a meaning and be constituted as objects of knowledge with and through their intra-actions with various other (non-) human actors (Højgaard and Søndergaard 2011), including the various discourses around technologies, RCTs and clinical practices. The in-hospital fall, the RCT and the bed sensor system studied here are, thus, phenomena simultaneously enacted and enacting. With the concept of intra-action, therefore, Barad asks us to focus on the phenomenon of in-hospital falls as the basic unit of our analysis.

While the sensor system attempts to reduce falls by alerting clinical staff to specific body movements (and the RCT attempts to trial this technology), our shift towards material-discursive practices means that reality is not 'out there' ready to be found by observing bodies through the use of 'objective' technologies of sensing and measurement. We can never step outside our phenomena of interest and measure them at a distance using representational processes, such as those of a RCT, thus avoiding "..a direct material engagement with the world" (Barad 2007: 49). The RCT attempts to structure, coordinate and make sense of standardised and comparable worlds (Timmermans and Epstein 2010). Both the RCT and the technology it is trialling are 'material-discursive practices' 
(Barad 2003: 818) which attempt to configure human and non-human actors, discourses, meanings and experiences. They 'cut' (in Barad's (2007) sense of the word) the prevention of in-hospital falls in specific and temporary boundary-making ways. In fact, these:

'...apparatuses are the material conditions of possibility and impossibility of mattering; they enact what matters and is excluded from mattering. Apparatuses enact agential cuts that produce determinate boundaries and properties of "entities" within phenomena, where "phenomena" are the ontological inseparability of agentially intra-acting components. That is, agential cuts are at once ontic and semantic. It is only through specific agential intraactions that the boundaries and properties of "components" of phenomena become determinate and that particular articulations become meaningful. In the absence of specific agential intra-actions, these ontic-semantic boundaries are indeterminate. In short, the apparatus specifies an agential cut that enacts a resolution (within the phenomenon) of the semantic, as well as ontic, indeterminacy' (Barad 2007: 148).

The analytical focus here is on the in-hospital fall not just as a problem that burdens healthcare and which is to be reduced by sensor system. Instead, the 'fall' (and its prevention) is a phenomenon of knowledge that is made and unmade through intra-actions between environment, culture, humans and technologies. The fall and its aversion are, thus, a phenomenon in situated practices and a representation of practice;

'Agential for the conceptualization that everything does something, that everything is performative and has agency-nothing is delimited, everything is always in intra-activity with something else, and Realism as the concept for the fact that the agentiality has real effects. Consequently, realism is not about representation of something substantialized or something that is already present as the difference between subject and object, between materiality and discourse. Realism is about real effects of intra-activity.' (Højgaard and Søndergaard 2011: 8-9) 
Barad's focus on accountability is also helpful here in exploring the ethical implications of "even the smallest cuts" (Barad 2007: 384) the sensor system and the RCT attempt to make and, thus, for the re-making of the acute care ward.

"We are responsible for the cuts that we help enact not because we do the choosing (neither do we escape responsibility because "we" are "chosen" by them), but because we are an agential part of the material becoming of the universe. Cuts are agentially enacted not by wilful individuals but by the larger material arrangement of which "we" are a "part." (Barad 2007: 178-79)

These apparatuses do not measure but, rather, produce material realities. We can study how the materialities of the RCT and the sensor system come to matter and how "objectivity is redefined in terms of accountability to the interferences made and the responsibilities following from intra-acting within and as part of the world" (Mjaaland 2017: 13). We analyse what boundary-making and marking they attempt to enact, what inclusions and exclusions they entail and what possibilities they configure, particularly in relation to those already present in the wards.

\section{Methods}

The RCT was of a conventional design, with patients on the wards studied randomised to either the intervention group (sensor) or the control group (no additional interventions, usual care). An intervention of this type could not be blinded, a limitation acknowledged by the researchers (Sahota et al. 2013). In addition to the main RCT, several methods of qualitative data collection were used. Qualitative, semi-structured interviews were carried out with nurses and Health Care Assistants (HCAs) $(n=20)$, and patients ( $n=18)$. Interviews took place on the wards being studied. Interviewers were trained and supervised by one of the research team. Interviews were digitally recorded and transcribed verbatim. Staff and patient interview data were initially analysed separately, through a process of coding and theory-building. This paper draws principally on the interview data, though it is informed by the other data collected. 
16 structured observations of the system in use on the wards were carried out on randomly selected weekdays. Observations took place in 5 -hour sessions (a total of 80 hours) throughout the day (07:00-12:00; 12:00-17:00 and 17:00-22:00 hours) to capture all non-sedentary patient activity (i.e. the waking hours). Observations focussed on the location and position of the patient, the response when the sensor alarm was activated, the response when the patient made a call, any activity or interaction, who was present, and what was heard and seen. The data were recorded by written notes made during and immediately after the sessions.

Ethical approval was granted by the relevant NHS Research Ethics Committee. All participants received written information in advance, and written consent was taken. Anonymity and confidentiality were assured.

\section{Results}

Data from patient interviews are reported separately below. The pressure sensors system was predicated on nurses responding to the radio pagers. Yet the data (all types) showed that nurses did not respond - or to be precise, responded selectively. The system was not used in the ways its designers intended, on a simple 'stimulus -response' model. It is important to emphasise that both the interview and observation data confirm that nurses were not uncaring or unconcerned about falls or safety generally. Staff used the system, but in a way that sought to minimise its 'intrusion' on the rest of their working practice. This manifested itself in several different ways:

Not carrying the bleep or delegating it 'down the line'

Registered nursing staff tended not to carry the bleep from the sensor system, claiming that "the trained staff have got duties to do [such] that they can't be disturbed" (Ward Manager). This meant that the bleep was often carried by HCAs. All of the HCAs who were interviewed confirmed that this was indeed the case. While this was not necessarily a problem, it does suggest that dealing with the sensor system was not viewed as a priority by the registered staff. The ward managers interviewed confirmed that "there tends to be ... reluctance" to carry the bleep. Likewise, "staff forgotten to hand 
over the pager to colleagues (Ob.8)". One interviewee was rather more emphatic: "No one wants to hold it. No- one!" (HCA).

\section{Technical problems with the system}

It is possible that one way that staff sought to minimise use of the system was by claiming that it was technically unreliable, or that faults were not corrected. Again, it is difficult to establish this objectively, but complaints about battery life on the bleeps were common.

they [batteries] don't last very long (Staff Nurse)

Other malfunctions attributed to the system included 'phantom' alarms:

RES: The sensor goes off on its own for whatever reason.

INT: While the bed is empty?

RES: While the bed is empty yes.

It is possible that this is a rhetorical device that enable staff to voice their complaints about the systems, and justify their reluctance to use it. Equally, it could be that a technology that appears to 'work' well in the lab is not robust enough to cope with rigours of life on a hospital ward. The observational data confirm this :

"non-functioning pager (Ob.3 \&.7)"

"faulty pagers [fault often due to the battery not being replaced] (Obs. $2,3,4)$ "

Sahota et al (2013) gives a figure of 120 system technical 'problems' over 25 months - our data would suggest that is an under-estimate.

Ignoring alarms from patients staff 'knew' to be either bed-bound, or 'healthy'

When asked about whether they ever ignore alarms, staff universally said that they did not. However, further discussion showed that there were times when alarms were apparently dealt with after a delay. Staff justified this in a variety of ways. For instance: 
... there is no chance of them standing up, I mean we've had it with bed-bound patients where the alarm was continually going off or people sitting in the chair fast asleep. (Staff Nurse)

In other words, the alarm could safely be ignored because the staff knew that the patient was immobile and thus incapable of getting up, and running the risk of falling. Similarly:

a patient, she was bed-bound so therefore she couldn't get up, but she was also rolling about on the bed, which was making the pager go off. (HCA)

By contrast, there were also those patients where alarms could be ignored, because the patient was viewed as being sufficiently healthy to be able to get out of bed safely by themselves.

another patient she was independent and she was taking herself to the toilet ... you just know that that patient is alright (HCA).

Ignoring alarms because they were busy with another patient

Another reason given for ignoring alarms, if only temporarily, was that the staff carrying the bleep were sufficiently busy with another patient that they could not get to the bed where the alarm had gone off. The following is typical:

I mean there are times when you can't get there, ... if I'm with a patient who's already fallen to the floor I can't physically go from that patient to the other patient, ... or I might be walking a patient to the toilet, ... and there's hardly any staff on the ward, say at night, sometimes we only have three staff on, you can't shout at night time, so it's going to be impossible for me to leave a patient who l'm walking (HCA)

Infection control was another reason given to justify a delayed response to a bleep:

If I am in the middle of like say cleaning up a patient that has been like sick or something, because of infection we try and get that done, or we'll say to the staff nurse I'm just busy, [bed number] has just gone off and someone will go and have a look. (HCA) 
Again, it is impossible to assess objectively how frequent these incidents were, or how widespread these practices were among the staff group. However, the fact that staff were prepared to discuss them within the context of a research interview suggests that they were reasonably common, and viewed as being relatively unproblematic by the staff themselves.

\section{$\underline{\text { Turning sensors off }}$}

This appears from the interview data to be a comparatively unusual, albeit not totally unheard of, practice.

INT: Have you ever switched [it] off?

RES: Yes, we have, when we've not had enough staff and we have written a note to say pager not been answered due to lack of staff, and we do, we only do that on very... it's been very rare occasions, but we always make it known that we haven't been able to do it because we've perhaps had a couple of people dying on the ward and we just have not had the manpower to answer them.

All of these issues with the system need to be understood in a wider context of the ubiquity of alarms in the modern hospital, and the fact that most alarms are, most of the time, ignored (Heath et al. 2003). As they show, this is because an alarm is usually the responsibility of one person, and can safely be ignored by others. It is possible that this learned behaviour of ignoring alarms has been transferred to the sensor system. There was some debate about the 8 second delay between the sensor being activated, and the alarm going off. It was characterised both as too long and not long enough. All of the above phenomena are instances where the nurses adapted the system or its usage to fit (or fit better) with their own, well-established patterns of working. The pattern is not one of outright refusal to use the system, but a more complex picture of selective and adaptive usage, so that the systems 'works' with their existing practice and ideas, where a variety of technologies and knowledges were already at work to prevent falls. 


\section{Justifying how the system was used}

The pattern of usage described above was justified in interviews with reference to ideas about how nursing work should be done.

well I think if it worked properly, if it was accurate ... and you'd got enough staff ... then it would be good because obviously it's highlighting when people are getting up (Deputy Sister)

I think if you've got enough staff to monitor it, it is a good idea (HCA)

It is impossible to establish the existence of staff shortages definitively, though at least one interviewee was sceptical. As Cohen (2011) shows these kinds of shortages can have very real effects for both nurses and patients. The possibility that there might be insufficient staff to use the bed sensor system safely was not anticipated by the research team, and, as such, no provision was made for it in the study. The observational data tended to confirm that staffing was an issue:

"it was evident that when the wards got busier at lunchtime, alerts were not reset promptly" (Ob $9,10,13)$

"Varied reasons were identified by the observer for prolongued and non-resetting sensor alerts and included:

- $\quad$ the staff were busy with other patients or activities (Ob.3 Ob.6),

- the wards being busy"

Criticism of the sensors, though common, was often nuanced, and expressed along with more supportive views:

If management are positive about it and support the staff with it then you know I think it will work. (Deputy Sister)

and

INT: $\quad$ So would you say that the sensor system has reduced the number of falls?

RES: Yes, it has. It has. (Staff nurse)

Though this critical view was shared by most interviewees, it was not universal. Some interviewees were positive about the sensors, and did not subscribe to the widely held critique of them: 
INT: So could you a little bit describe your experience of the sensor, what do you think works well and not so well, advantages, disadvantages?

RES: I think that they are good, I really do think they are good. (Deputy Sister)

Indeed, one interviewee thought that numbers of staff were not an issue.

INT: And there's always someone around?

RES: There's usually somebody around you know (Ward Sister)

The seniority of the staff interviewed appeared to be a factor in their level of enthusiasm for the system. While most interviewees were consistent in their position on the system, two discussed that their views had changed during the project, from an initial scepticism to a more enthusiastic stance. The scepticism was rooted in ideas about how nursing practice should be done, particularly ideas about how patients who were at risk of falling should be cared for. It was this mismatch between the ideas about falls and fallers inherent in the sensor system (and the RCT), and nurses' own ideas about them, well established in nursing culture and practice, that was central to understanding the fate of the sensor system and the RCT. Falls were already acknowledged as an issue, both by the hospital, and the nurses themselves, and well-developed systems were in place to address it. The sensors were thus a disruptive element in this rich assembly of policy, practice and technology.

\section{Falls and fallers}

Despite understanding the principles of the RCT methodology, nurses persisted in wanting to override the random allocation of patients to sensors (or control group). A commonly held view among the staff was that they knew which patients were at risk of falling, and which were not (a view supported by research evidence (Meyer et al. 2009), and they should be allowed to determine whether a patient received a sensor or not.

Whether they'd work better when we can choose who they go on, because it's a trial and you know it's blind isn't it, so they're just putting it on anybody, then we've not, you know 
we've not identified a patient that we think needs it, so whether it would be different then or not. (Ward Sister)

when the time comes when we can choose who has the sensor then that would be better. (Staff Nurse)

It seemed paradoxical to nurses that, due to the randomisation process, patients who were clearly not in need of a sensor would be allocated one, not least when the allocation contradicted their own expertise. . Some interviewees said that this was an inevitable consequence of the methodology of the RCT, but others continued to find it incomprehensible.

The RCT and the system made unacknowledged assumptions about the nature of a fall, and how they were reported. When asked, all interviewees claimed that all falls are reported, no matter how trivial. However, this was not always the case.

RES: Every single fall should be reported.

INT: Should be reported?

RES: Yes.

INT: Has the trial changed this, this sensor study, changed this practice?

RES: Yes, I think everybody is a lot more aware of it. Yeah, I think it's raised a lot more awareness, yeah. (Staff Nurse)

The implication of this exchange being that the introduction of sensors was thought to have increased the reporting of falls, suggesting that not all falls had been reported in the past. There were also suggestions that what counts as a fall is subject to a degree of interpretative flexibility, with interviewees using terms like slip or trip to distinguish these incidents from 'falls'. This is analogous with the findings of Walker, Porock and Timmons (2011) who also found that the concept of a fall is flexibly interpreted. Falls were viewed by some interviewees as inevitable. This interviewee sought to distance themselves from that view, but claimed it was widespread: 
I think you've got to change the idea of what the nurses think about the fall, the majority of them think well with elderly and there's some type of people you're going to get a fall, no matter what you do. (HCA)

We would argue that here is another instance of the system producing ideas about practice that were not shared by the nurses who used the system. The system and the RCT are predicated on a notion that falls are a homogeneous event, and that they are preventable. These ideas are not the same as those held by the nurses, who that that falls were, to a degree, inevitable. .

\section{Patients}

A number of the patients interviewed were unaware of the sensor, despite all have given consent to take part in the wider RCT. The interviews with patients covered a wide range of topics, driven as they were by the concerns of the patient interviewees, and what is perhaps most striking about this data is how little the patients talked about the sensor system. Some did, however, describe activity apparently relating to the use of sensors on the ward:

...sometimes when nurses come in and they were talking to the patient as though they've been called. (Patient 14)

It is clear that many nurses did not include patients in the routine use of sensors, as it was rarely communicated to the patient the reason why a nurse had been 'called to the bedside'.

"There was very limited nurse-initiated interaction between nurse and patient on resetting the sensor alert" (ob.3)

Patients who were aware of the sensor sometimes limited their movement in order to avoid activating their sensor. They described feeling embarrassed if a nurse came into the room after the sensor had been activated unintentionally. Four patients made comments which suggested that they believed that they had been selectively chosen to be in a particular group. Whilst some people felt 
reassured as a result of having the sensor, some in the non-intervention group were grateful that they had been considered 'well enough' not to require a sensor.

They should give them to old people that keep getting up you know, I think they deserve them more than I do you know because I'm pretty careful ... so I think somebody else deserves it more than me (Patient 5).

The staff reported that some patients disliked the system sufficiently to ask to have it removed:

We've had a few where they've asked for them to be taken off because it has actually annoyed them. We've had a couple like that (Nursing Assistant)

Something that was not reported in the patient interviews, but the staff claimed had happened was the patients either deliberately setting the alarm off in order to get attention, or resetting or turning the system off themselves.

RES : $\quad . .$. they sense that if their buzzer goes off they will get attention.

INT: $\quad$ So they use it as a way to get attention? Do they use it as a...?

RES: $\quad$ But then for others who actually unplugged it!

INT: They found a way to unplug it?

RES: $\quad$ Because it gets on their nerves, yeah. (Ward Sister)

And

"patient tried to disconnect the sensor (Ob. 14)"

Patients were reported to have justified this in terms of not wanting to bother the nurses:

you'll find that she's thrown the thing on the floor because ... every time that goes off you have to come and you're busy. So some patients are aware that every time they move that alarm goes off, which then means the nurse or whoever has got to come and switch it off, and they're aware that we're busy and they feel like they're being a nuisance. (HCA) 
This phenomenon was also reported to the observer (Ob. 5).

\section{Discussion}

We used Barad's theoretical framework of agential realism, to show that there is no objective, epistemologically independent, body movement (that might cause a fall) that the sensors had to detect and alarm for. This technology had to articulate in its design and use the ontological practice of falling as experienced by elderly patients and nurses within a hospital ward. Body movement was but one of the many highly complex intra-actions and practices that had to be in place for nurses to avert the falls. But even the linking of every body movement to an attempt to get up jeopardised the specificity and fidelity of the two apparatuses studied here (sensors and RCTs) to detect the (reduction in) falls. The intra-active agential cuts of the RCT and the sensor system, as boundarymaking practices, enacted certain inclusions, exclusions and assumptions of what matters, and thus should materialise, for the measurement of the effectiveness of technology-mediated aversion of falls. Specifically, it was only when the phenomenon of interest was enacted, that is, the patient moved enough to trigger the sensor, that the RCT and the technology resurrected and acknowledged the subjectivity and agency of the radio-pager equipped nurse (an otherwise passive object waiting to be called upon as and when needed) and the patient at risk (a passive object waiting to fall). Both of these apparatuses attempted, unsuccessfully, to individualise the 'response-ability' (Schrader 2010) to prevent a fall away from the ward (including the patient), and from the other 'confounding' environmental and contextual (f)actors intra-acting with each other, to only those who carried the pager.

The need for objective measurements on the part of the scientific team, particularly in relation to the random allocation of these sensors, without input from the nurses on which patients might be appropriate, contributed to the failure of this technology to show evidence of reducing falls. It allowed nurses and patients to ignore alarms or even de-activate the sensors. The RCT, as a scientific performativity, sought to control all 'extraneous' factors that can be found in a hospital ward via 
randomisation in order to establish causality from intervention to outcome. However, as we have shown, neither the intervention (the sensor system), nor the outcomes (falls) could be disentangled from the broader social context of the ward, the nurses and their work. In effect, the nurses reallocated patients within the RCT by their varied (albeit rational) responses to the alarms. Rather than the trial participants studied by Featherstone and Donovan (2002) who effectively re-allocated themselves within an RCT, this is an instance of the patients being re-allocated by clinical staff. The sensors as an apparatus for averting falls were entangled with the apparatus of the RCT. Both apparatuses attempted to impose their own indeterminacies and exclusions that failed to reproduce the material-discursive reality of falls to be measured as they were coming into conflict within those of the hospital ward. The clash of the agential cuts and routine practices of both the RCT and clinical care performed here prohibited the reading and aversion of the phenomenon of inhospital falls "...with a potential knock-on effect for patients and subsequently for recruitment and retention in the trial" (Montgomery 2017: 28).

Firstly, we argue that the bed or chair, the patient (with her body) and the nurse (carrying the radiopager) were treated as taken-for-granted entities with specific properties and a priori boundaries that the technology had then to represent and bring together in order to avert the falls. However, within the hospital ward, these essentialist assumptions were disturbed and destabilised. From an agential realist perspective, the design of a sensor should not be just about the sensing of body movement and, thus, about alarming for a fall after a specific delay waiting for a response by a person. It should instead attempt to represent the practice of fall aversion or the awareness and response to the falling patient by clinical staff, to the extent that it can prevent it from happening in a hospital ward. The aim here is to move away from discussions of subjects and objects, or which actor failed to complete the script, but to understand, examine and articulate the phenomenon of falling (as the basic unit of analysis) into the new socio-technical practice. The hospital fall here is a distinct type of fall, rather than a pre-given reality independent of the setting and material-semiotic practices which allow its performativity to take place. This fall is not like any other fall out 'there' 
that it is just being transferred onto-epistemologically into the hospital. Rather than an ontological truth, where the sensors await its materialisation so as to pick it up and produce the alarm, then, the fall should have been treated as a phenomenon to be experienced by staff and patients through multiple and complex intra-actions.

The introduction of the sensor in a hospital ward required a whole range of re-ordering of practices while introducing new ones. It required a range of actors and new intra-actions to be constantly performed around this new technology-in-trial. While it might have raised awareness of falls (based on the qualitative data), it introduced a homogeneous definition of what constituted an in-hospital fall, which was not locally agreed and did not differentiate (for nurses) from a slip or a trip (again, at variance with nurses' own, well-developed, ideas and expertise). It also did not account for the inevitability of these falls for each acute care ward or for which specific patients at risk this technology was more appropriate, given the available human resources and the allocations of nurses among the three shifts during the day. It is these sociomaterialities that the bed sensor system also had to re-produce so as to monitor falls and alarm staff accordingly.

Moreover, it effected specific times and spaces which had to find a way to intra-act with those of the hospital ward. It assigned new responsibilities and control to specifically configured docile users of this technology (Akrich 1992), for example, to staff who were required to carry the radio-pager with them at all times. However, the RCT design, perhaps more than the technology itself, assumed that the radio-pager-equipped nurse/HCA would always be available to respond rapidly, and consistently, irrespective of the geographies of the wards and regardless of whatever other work she might be doing. As we have shown, the nurses and HCAs had other work to do, that (at times) they viewed as being of a higher priority than the sensor system. Rather than use the technology in the way the designers intended, the nurses developed their own ways of using it (or not using it) that they perceived as better fit with their existing working practices. A good example of this is the delegation of the role of 'bleep-holder' from senior to more junior staff (McLaughlin and Webster 1998). The RCT design, perhaps at the expense of the technology, reproduced professional hierarchies and 
made the response to an alarm a personal matter of an individual member of staff, instead of a collective effort for the whole ward which was not how the responsibility was understood by nurses. Even though the sensor system might be assumed to be a priority for nurses, designed as it was to protect patients from falling, it did not conform to the nurses' ideas about how they worked. They regularly had more pressing priorities, in terms of direct patient care, than attending to the system alerts. This was also exacerbated by the fact that the nurses did not agree with the risk assessments that had effectively been made by the system. As discussed above, they perceived that there were patients who had bed sensors who were at little or no risk of falling, while other patients were more at risk. Similarly, the nurses interviewed had clear ideas about how wards 'should' be staffed, that is, what the appropriate number of staff and skill-mix should be in order to care for patients as they saw fit.

In that context, even the properties of the sensor itself with its limited battery life, malfunctioning (see also Sahota et al, 2013), the 8-second delay in alarming the staff and the ease with which it could be de-activated by patients and staff, proved to be problematic in its attempt to sense, alarm for and measure the fall - a phenomenon ascertained from incident reporting forms completed by the ward teams (Sahota et al 2013). In addition to caring for patients and averting falls, nurses had also to 'care' for the radio-pagers to ensure their continuous operation (e.g. changing and charging batteries). The 8-second delay was, for the ward, an agential cut only for a specific type of patients and their body movements, since this alarm delay might have been enough for some patients (but not for others) to avert the fall. Depending on the circumstances, it could notify staff that some patients were, indeed, about to fall off the bed or chair while, for other patients, that they had already fallen (Shorr et al. 2012). The design of the system (and its agential cut) was predicated on a 'docile' patient who lay flat and still in the bed. Some patients did not conform to this configuration, hence there were the 'false' alarms. In some cases, it even restricted patients in their movement in bed so as not to trigger the alarm 'unnecessarily'. Here, a fall prevention technology, instead of facilitating safe mobility, became a restraining device, which could have caused other complications 
associated with immobility during hospitalisation (e.g. pressure sores) (Inouye et al. 2009), an example of an onto-ethical-epistemological issue (Barad 2007).

It required patients to subjectify themselves as at a monitored risk of fall. Apart from succumbing to the material-semiotic enactments that constitute an acute, medical elderly care ward, these patients (and nurses) had to accept the sensor. This was, in some cases, resisted and instead of being accepted as a technology to keep them safe from falling, the sensor system was enacted as a communication device through which nurses and patients could intra-act for purposes beyond the trialling of the system. The technology became flexible enough to be used as a draw-attention device or even the opposite, when it was de-activated by those patients that wanted to be left alone or did not want to be seen as 'demanding' patients 'wasting' nurses' time for no reason. This could have jeopardised the safety of these patients if clinical staff relied solely on the sensor to prevent falls.

\section{Conclusion}

The performative approach of Barad's agential realism, with its focus and analytical lens on new subjectivities, materialities, discourses, spaces, times and movements, as effects of intra-actions and agential cuts, proved to be an insightful way of studying a patient safety technology implementation in clinical practice. It opened up new discursive spaces in order to include in the qualitative evaluation of an RCT - and by extension in the design, application and use of this technology - the experiences and voices of those who, in this case, had to carry radio-pagers and respond to alarms as well as of those of patients who stay in bed with them. It also raised the wider question of what kind of new subjectivities, agencies and power relations these intra-actions with technologies might effect and (re-)produce in the hospital ward that facilitate or obstruct patients' communication of their needs to the clinical staff and how these might (unjustifiably) be seen as a nuisance and 'waste' of clinical time. While our study was restricted to interviews and observations, we were able to demonstrate the value of social sciences working systematically alongside RCTs, instead of leaving 
explanations of failure as assumptions and notes of reflexivity in the 'Discussion' section of an RCT paper.

\section{Acknowledgement}

This research was funded by the National Institute for Health Services Research (NIHR)-Research for Patient Benefit Programme (RfPB)-PB-PG-0107-11112. The views expressed are those of the author(s) and not necessarily those of the NIHR or the Department of Health and Social Care.

\section{References}

Akrich, M. (1992) The De-Scription of Technical Objects. In Bijker, W., Law, J. (Eds.) Shaping technology/Building society studies in sociotechnical change, Cambridge, MA: MIT Press, pp. 205-24.

Allen, D. (1997)The nursing-medical boundary: a negotiated order? Sociology of Health and IIIness 19(4) 498-520

Barad, K. (2003) Posthumanist performativity: toward an understanding of how matter comes to matter, Signs: Journal of Women in Culture and Society, 28, 3, 801-31.

Barad, K. (2007) Meeting the universe halfway, London, UK: Duke University Press.

Bijker, W., Hughes, T. and Pinch, T. (eds.) (1987) The Social Construction of Technological Systems: New Directions in the Sociology and History of Technology, Cambridge, Mass.: MIT Press

Cohen, R. (2011) Time, space and touch at work: body work and labour process (re)organisation, Sociology of Health \& Illness, 33, 2, 189-205.

Featherstone, K. and Donovan, J.L. (2002) "Why don't they just tell me straight, why allocate it?" The struggle to make sense of participating in a randomised controlled trial, Social Science \& Medicine. 55, 5, 709-19.

Heath, C., Luff, P. and Svensson, M. S. (2003) Technology and medical practice, Sociology of Health and IIIness, 25, 3, 75-96.

Højgaard, L. and Søndergaard, D.M. (2011) Theorizing the complexities of discursive and material subjectivity: Agential realism and poststructural analyses, Theory \& Psychology, 21, 3, 338-54.

Horton, S., Mares, K., Coull, N. and Poland, F. (2017) On the character and production of 'active participation' in neuro-rehabilitation: an Actor - Network perspective, Sociology of health \& illness, 39, 8, 1529-41.

Inouye, S.K., Brown, C.J. and Tinetti, M.E. (2009) Medicare non-payment, hospital falls, and unintended consequences, New England Journal of Medicine, 360, 23, 2390-93.

Latour, B. (2005) Reassembling the Social: An Introduction to Actor-Network Theory, Oxford: Oxford University Press. 
McLaughlin, J. and Webster, A. (1998) Rationalising knowledge: IT systems, professional identities and power, The Sociological Review, 46, 4, 781-802.

Meyer G, Köpke S, Haastert B, et al. (2009) Comparison of a fall risk assessment tool with nurses' judgement alone: a cluster-randomized controlled trial, Age and Ageing, 38, 417-23.

Mjaaland, T. (2017) Imagining the Real: The Photographic Image and Imagination in Knowledge Production, Visual Anthropology, 30, 1, 1-21.

Mol, A. (2002) The Body Multiple: Ontology in Medical Practice, Durham, NC: Duke University Press.

Montgomery, C.M. (2017) Clinical Trials and the Drive to Material Standardisation, Science \& Technology Studies, 30, 4, 30-44.

Montgomery, C.M. and Pool, R. (2017) From 'trial community' to 'experimental publics': how clinical research shapes public participation, Critical Public Health, 27, 1, 50-62.

Riemer, K. and Johnston, R. (2014) Rethinking the place of the artefact in IS using Heidegger's analysis of equipment, European Journal of Information Systems, 23, 3, 273-88.

Savransky, M. and Rosengarten, M. (2016) What is nature capable of? Evidence, ontology and speculative medical humanities, Medical Humanities, 42, 3, 166-72.

Schrader, A. (2010). Responding to pfiesteria piscicida (the fish killer) phantomatic ontologies, indeterminacy, and responsibility in toxic microbiology, Social Studies of Science, 402, 275-306.

Till A, Sahota O, Piper J, Grainge MJ (2005) Bed Sensors Reduce In-patient Falls and Hospital Length of Stay Age and Ageing 2005,34(Supplement 2):ii15

Sahota O. (2009) Vitamin D and in-patient falls. Age and Ageing. 2009;38:339-40

Sahota O. et al (2013) REFINE (REducing Falls in In-patieNt Elderly) using bed and bedside chair pressure sensors linked to radio-pagers in acute hospital care: a randomised controlled trial. Age and Ageing 43(2): 247-253

Timmermans, S. and Epstein, S. (2010) A World of Standards but not a Standard World: Toward a Sociology of Standards and Standardization, Annual Review of Sociology, 36, 69-89.

Timmons, S. (2003) Nurses resisting information technology. Nursing inquiry 10 (4), 257-269

Vassallo, M., Poynter, L., Sharma, J., Kwan, J. and Allen, S. (2008) Fall risk-assessment tools compared with clinical judgment: an evaluation in a rehabilitation ward, Age and Ageing, 37, 3, 27781.

Walker, W., Porock, D. and Timmons, S. (2011) The importance of identity in fall prevention. Nursing Older People 23, 2, 21-28.

Webster, A. (2002) Innovative Health Technologies and the Social: Redefining Health, Medicine and the Body, Current Sociology, 50, 3, 443-57. 
Will, C.M. (2009) Identifying Effectiveness in "'The Old Old"': Principles and Values in the Age of Clinical Trials, Science, Technology, \& Human Values. 34, 5, 607-28.

Will, C. and Moreira, T. (2016) Introduction Medical Proofs, Social Experiments: Clinical Trials in Shifting Contexts. In Will, C. and Moreira, T. (eds.) Medical Proofs, Social Experiments. Routledge. pp. 1-13.

Wohlwend, K.E., Peppler, K.A., Keune, A. and Thompson, N. (2017) Making sense and nonsense: Comparing mediated discourse and agential realist approaches to materiality in a preschool makerspace, Journal of Early Childhood Literacy, 17, 3, 444-62.

Woolgar, S. (1991) Configuring the User: the Case of Usability Trials. In Law, J. (ed.) A Sociology of Monsters: Essays on Power, Technology and Domination. London: Routledge. 